\title{
Ultra-Wideband Printed-Circuit Array Antenna for Medical Monitoring Applications
}

\author{
Hung-Jui (Harry) Lam \\ Wireless 2000 RF\&UWB Technologies Ltd. \\ 2421 Alpha Avenue \\ Burnaby, B.C., V5C 5L2, Canada \\ hlam@wireless2000.com
}

\author{
Jens Bornemann \\ Department of Electrical and Computer Engineering \\ University of Victoria, PO Box 3055 \\ Victoria, B.C., V8W 3P6, Canada
}

\begin{abstract}
A new ultra-wideband printed-circuit antenna structure for medical monitoring purpose is introduced. It consists of one single transmitting antenna and an array of receiving antennas in coplanar waveguide technology. In the transmit mode, the frequency of operation is between $3.4 \mathrm{GHz}$ to 5.9 GHz. The receiving antenna array operates from $3.5 \mathrm{GHz}$ to 4.8 GHz. Both bands show an input return loss better than $10 \mathrm{~dB}$. Radiation patterns for both transmitting antenna and receiving array are presented at selected frequencies. The analysis method is verified against previous measurements.
\end{abstract}

Keywords - Printed-circuit antennas, ultra-wideband antennas, (UWB), antenna array, coplanar technology, medical monitoring.

\section{INTRODUCTION}

In the recent rapid research of ultra-wideband (UWB) technology, the UWB antenna is one of the most essential components for a UWB system. Many applications such as local network, wireless communication and imaging radar employ UWB technology. UWB radars are used in various fields at short range for detection of an object's movements. An example in medical applications would be to detect a person's heart and respiration rates [1] - [3]

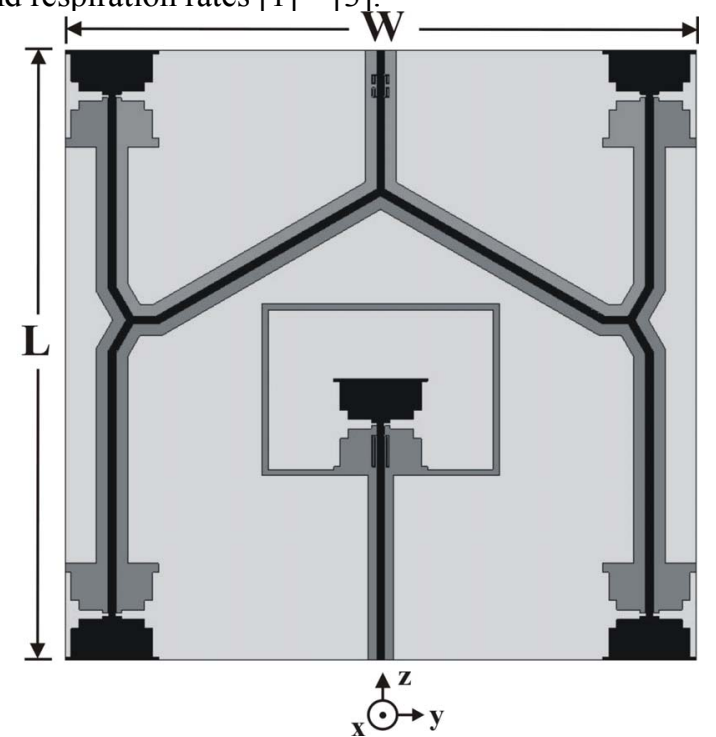

Figure 1. UWB antenna structure with a single Tx antenna and a Rx antenna array in coplanar waveguide technology.
Wireless 2000 has developed a Patient Assessment Monitor $\left(\mathrm{PAM}^{\mathrm{TM}} 3000\right)$ system which allows bed occupancy and vital signs monitoring of patients in elder care and health care facilities. The UWB antenna structure in Fig. 1 is designed to operate under the same specification as the UWB antenna in the PAM ${ }^{\mathrm{TM}} 3000$ but with several advantages. The operation of this UWB antenna structure is based on the Tx antenna transmitting the UWB signal and the $\mathrm{Rx}$ antenna array receiving the reflected UWB signal from movements of a patient's heart and thorax. The presented antenna in coplanar waveguide technology has a single transmitting antenna at the center and four receiving antennas at the corners.

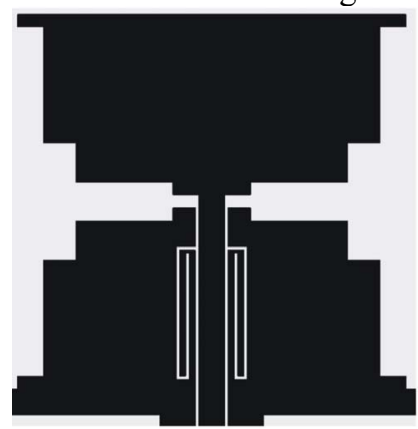

(a)
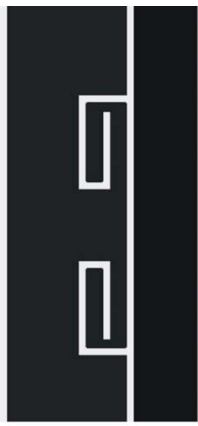

(b)
Figure 2. Notch filters in UWB antenna structure: a) the Tx antenna (center of Fig. 1) with the $2.4 \mathrm{GHz}$ coplanar notch filter, b) $6.6 \mathrm{GHz}$ coplanar notch filter at the feed of the Rx antenna array (top of Fig. 1).

Coplanar technology offers a number of advantages, e.g. [4] - [9], for the fabrication of printed-circuit UWB antennas. The coplanar transmission lines extend to edges (top for $\mathrm{Rx}$ and bottom for Tx in Fig. 1) which make the feeding much easier. The 180 degree phase shift between the top two receiving antennas and the bottom two receiving antennas is compensated by the half-wavelength difference in the transmission line at $4 \mathrm{GHz}$. Around the transmitting antenna, thin strip ground lines are introduced to limit reflection effects from the receiving antenna array. For coplanar waveguide transmission lines of the receiving antenna array, grounds are connected through via holes and back ground strip lines at each $\mathrm{T}$-junction. In both Tx antenna and Rx antenna array, coplanar bandstop filters (Fig. 2) are introduced to create a notch at selected frequencies. Similar concepts, with the same type of technology, have been applied to single element UWB 
antennas, e.g. [10], [11]. By following the basic concept of [12], a $2.4 \mathrm{GHz}$ bandstop filter for the Tx transmission line and a $6.6 \mathrm{GHz}$ bandstop filter for the $\mathrm{Rx}$ transmission line are created to improve the bandwidth performance for both the Tx antenna and Rx antenna array (Fig. 2). The design of the single element UWB antenna for the proposed UWB antenna structure follows basic principles outlined in [13]. In order to obtain the final result, several technical design concepts are followed. First, the transmitting antenna and the $2.4 \mathrm{GHz}$ bandstop filter are designed and optimized to achieve the accepted performance. With strip ground lines around the transmitting antenna, the design of the transmitting antenna can be performed independent of that of the receiving antenna array. Finally, the receiving antenna array and the $6.6 \mathrm{GHz}$ bandstop filter are designed and optimized to achieve the optimum performance. Most of the optimization is devoted to varying the length and width of strip ground lines around the transmitting antenna, and both bandstop filters' position and length in the transmitting antenna and the receiving antenna array.

\section{VALIDATION}

The design of the proposed UWB antenna structure is simulated and computed with the finite-element full-wave field solver software HFSS. With any simulation software, a major concern is validation of results. As shown in Fig. 3, input return loss simulation results of the UWB antenna presented in [11] are compared with measurements. Good agreement over the entire $2-11 \mathrm{GHz}$ bandwidth is observed, thus validating the design strategy in an HFSS environment.

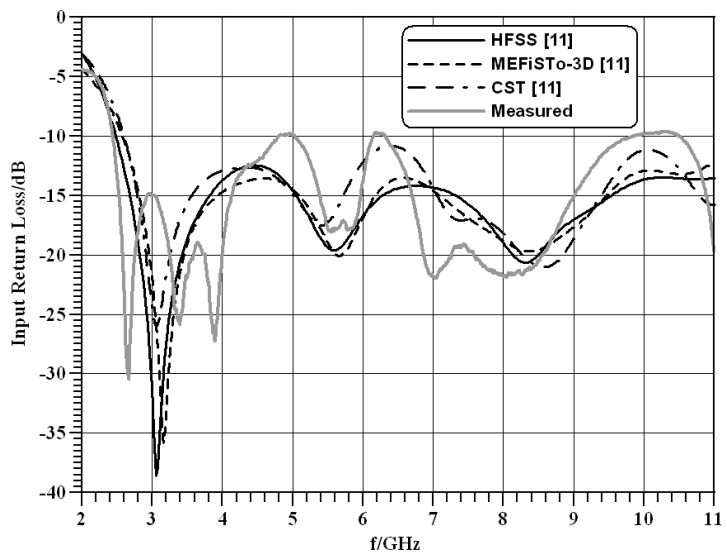

Figure 3. Input return loss performance comparison: simulation software results [11] and the measurements.

\section{RESULTS}

The ultra-wideband printed-circuit antenna structure in coplanar technology shown in Fig. 1 uses an FR4 substrate of $1.575 \mathrm{~mm}$ thickness and $202 \mathrm{~mm} \times 195 \mathrm{~mm}$ (W x L) substrate area. The permittivity parameters are $\varepsilon_{\mathrm{r}}=4.4$ and $\tan \delta=0.018$. The proposed UWB antenna structure is designed and simulated under the environment as close as possible to the UWB antenna in the PAM ${ }^{\mathrm{TM}} 3000$ system. Therefore, in the simulation model, a perfect E-plane, which acts as a ground metal reflector, is placed at the back of the UWB antenna structure. This ensures that all radiation go upward (positive $\mathrm{x}$ direction) because the UWB antenna structure is designed to be placed underneath a patient's bed. In both transmit and receive mode, models of coaxial cable are created in the simulation model for feeding purpose.

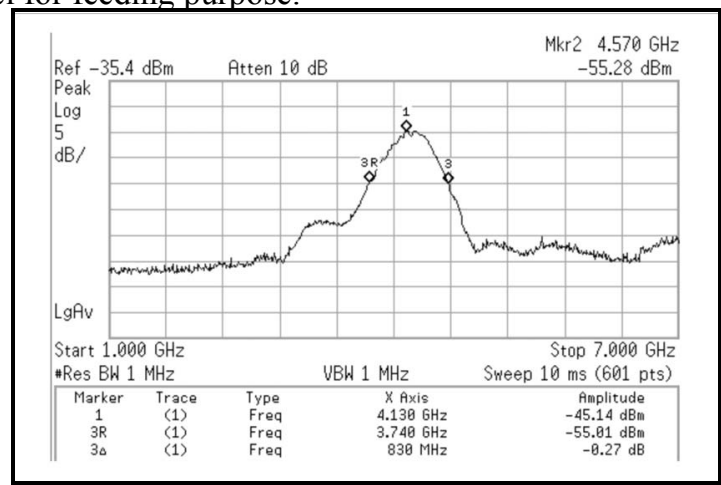

Figure 4. Spectrum of the generated UWB signal with $830 \mathrm{MHz}$ bandwidth

\section{A. Transmitting Antenna}

Fig. 4 shows the spectrum of the UWB signal that is used for the vital signs monitoring (before the transmitting antenna). According to FCC Part 15, the maximum allowed average power spectral density within this band is $-41.3 \mathrm{dBm} / \mathrm{MHz}$ for medical imaging. The generated UWB signal has a maximum average power spectral density of $-45.14 \mathrm{dBm} / \mathrm{MHz}$ at 4.130 $\mathrm{GHz}$ center frequency. The bandwidth of the UWB signal is measured at points that are $10 \mathrm{~dB}$ down from the maximum. In this case, the bandwidth of the generated UWB signal is 830 $\mathrm{MHz}$.

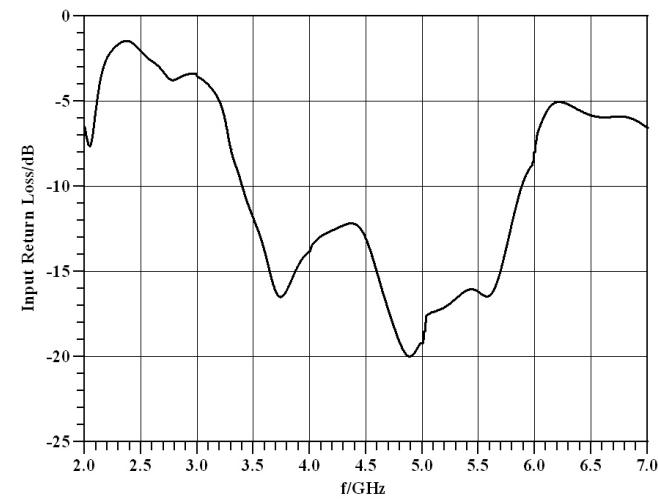

Figure 5. Input return loss performance of the UWB transmitting antenna shown in Fig. 1.

Based on the above description of the UWB signal, the behavior of input return loss for the transmitting UWB antenna plays a crucial role in the UWB transmit mode. If the input return loss has values greater than $10 \mathrm{~dB}$ at the center frequency of the generated UWB signal compared to the input return loss of the rest of the operating band, then the bandwidth of the transmitted UWB signal will be limited. The transmitting antenna will filter the generated UWB signal resulting in a narrower transmitted signal bandwidth. This is because the transmitting antenna will increase the maximum average power spectral density of the generated UWB signal relative to the rest of the operating band. Fig. 5 demonstrates that the input return loss between $3.4 \mathrm{GHz}$ to $5.9 \mathrm{GHz}$ is better than $10 \mathrm{~dB}$ for the proposed UWB transmitting antenna. Also, within the 
operating band, the maximum input return loss is not greater than $10 \mathrm{~dB}$ compared to the input return loss of the rest of the operating band. Thus, the transmitting antenna will not limit the bandwidth of the transmitted UWB signal but widen it by flatting the response of the average power spectral density of the generated UWB signal.

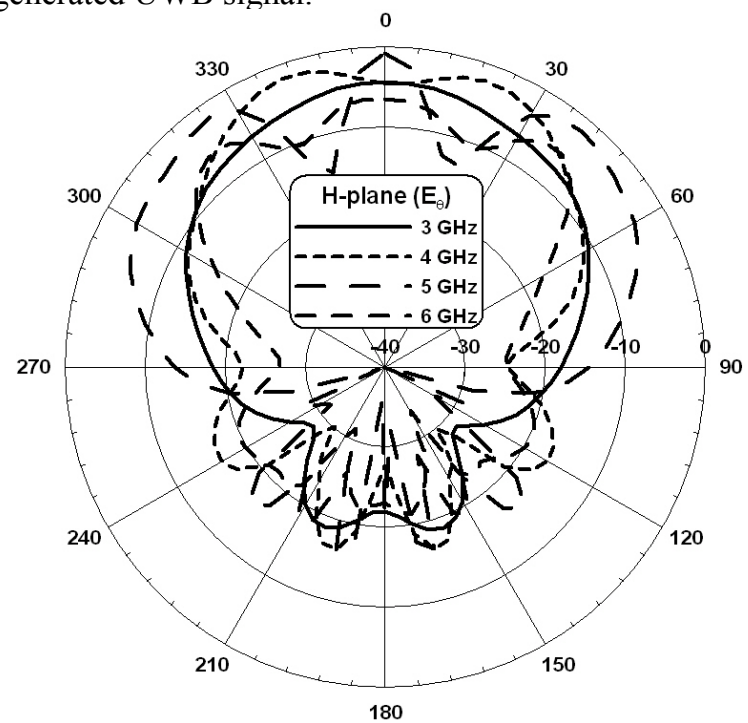

Figure 6. Normalized co-polarized H-plane (xy-plane) radiation patterns $\mathrm{E}_{\theta}(\pi / 2, \phi)$ of the UWB transmitting antenna for various frequencies.

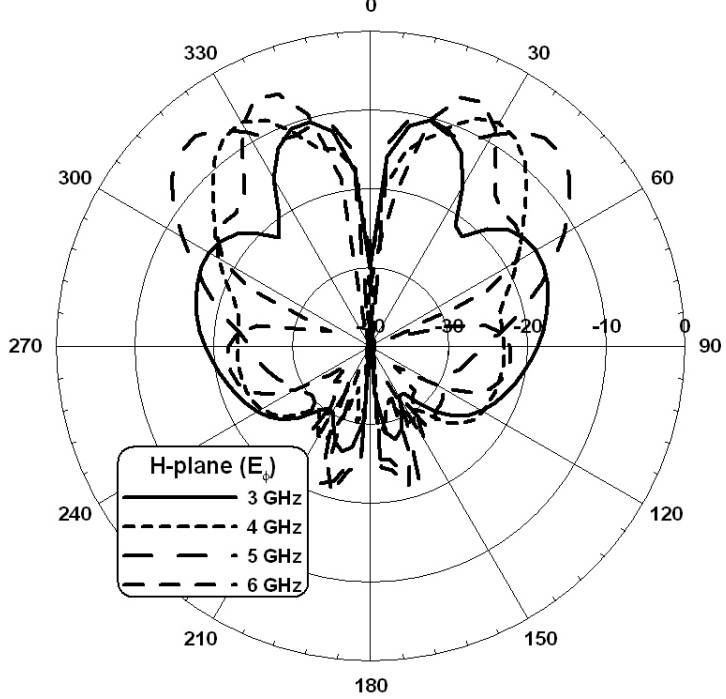

Figure 7. Normalized cross-polarized H-plane (xy-plane) radiation patterns

$\mathrm{E}_{\phi}(\pi / 2, \phi)$ of the UWB transmitting antenna for various frequencies.

To display the radiation patterns, the conventional definition of angles $\theta$ and $\phi$ with respect to coordinates $\mathrm{x}, \mathrm{y}, \mathrm{z}$ $(\mathrm{x} / \mathrm{r}=\sin \theta \cos \phi, \quad \mathrm{y} / \mathrm{r}=\sin \theta \sin \phi, \quad \mathrm{z} / \mathrm{r}=\cos \theta)$ is used. Radiation patterns are normalized to the peak gain of selected frequencies. The normalized H-plane radiation patterns in the xy plane (c.f. Fig. 1) are shown in Fig. 6 and Fig. 7. For both vertical polarization (Fig. 6) and horizontal polarization (Fig. 7 ), radiation patterns are pointing upward (positive $\mathrm{x}$ direction) as expected. In the vertical polarization (Fig. 6), in-band frequencies, at $4 \mathrm{GHz}$ and $5 \mathrm{GHz}$ tend to have greater gain values compared to $3 \mathrm{GHz}$ and $6 \mathrm{GHz}$ which are outside of the operating band. The reception in horizontal polarization, which is shown in Fig. 7 is due to the direction of the field in the coplanar feed line. As a result, these patterns are almost zero in $\phi=0$ degrees and $\phi=180$ degrees.

The co-polarized and cross-polarized patterns in the Eplane (xz plane) are shown in Fig. 8 and in Fig. 9. Again, both vertical (Fig. 8) and horizontal polarizations (Fig. 9) are radiated upward (positive $\mathrm{x}$ direction) as expected in the Eplane.

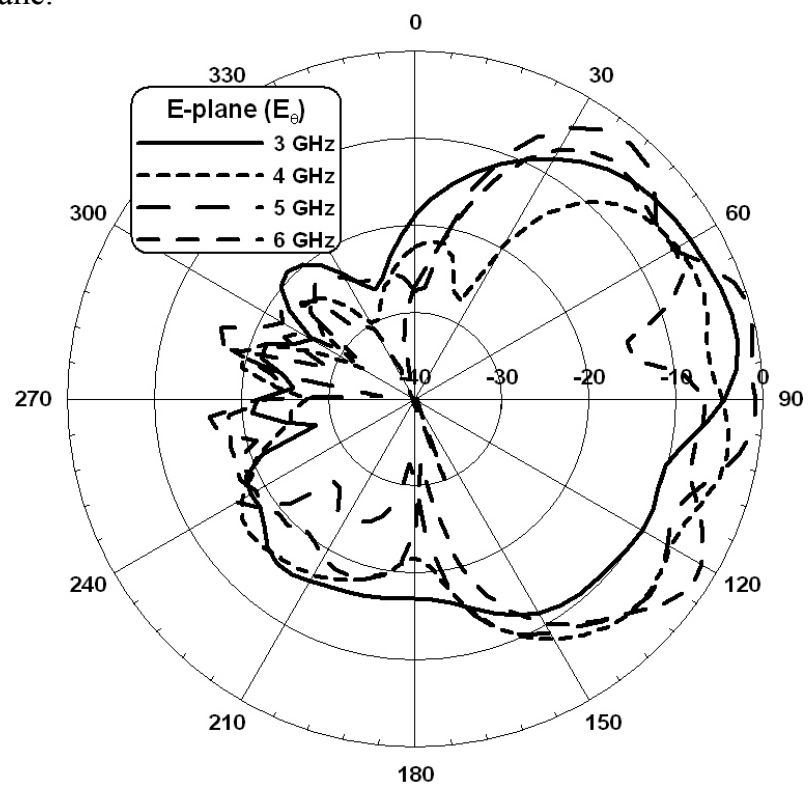

Figure 8. Normalized co-polarized E-plane (xz-plane) radiation patterns $E_{\theta}(\theta, 0)$ of the UWB transmitting antenna for various frequencies.

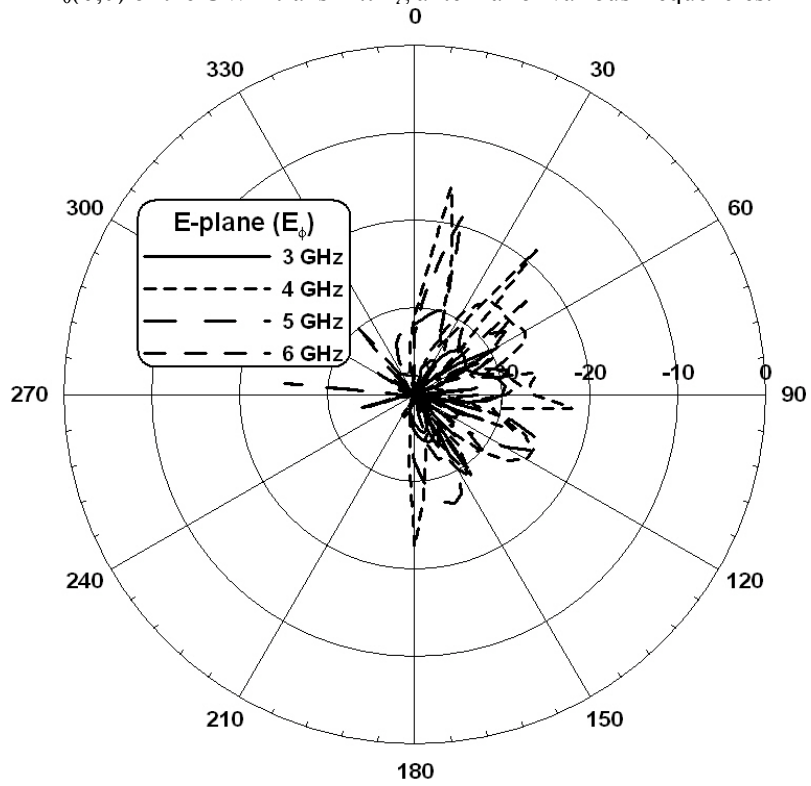

Figure 9. Normalized cross-polarized E-plane (xz-plane) radiation patterns $\mathrm{E}_{\phi}(\theta, 0)$ of the UWB transmitting antenna for various frequencies.

\section{B. Receiving Antenna Array}

The reason of using an antenna array in the receive mode is to increase the received coverage area for the reflected UWB signal from movements of a patient's heart and thorax. Therefore, the antenna array is not designed to be more directive than a single element. Fig. 10 illustrates that the input 
return loss between $3.5 \mathrm{GHz}$ to $4.8 \mathrm{GHz}$ is better than $10 \mathrm{~dB}$ for the proposed UWB receiving antenna array.

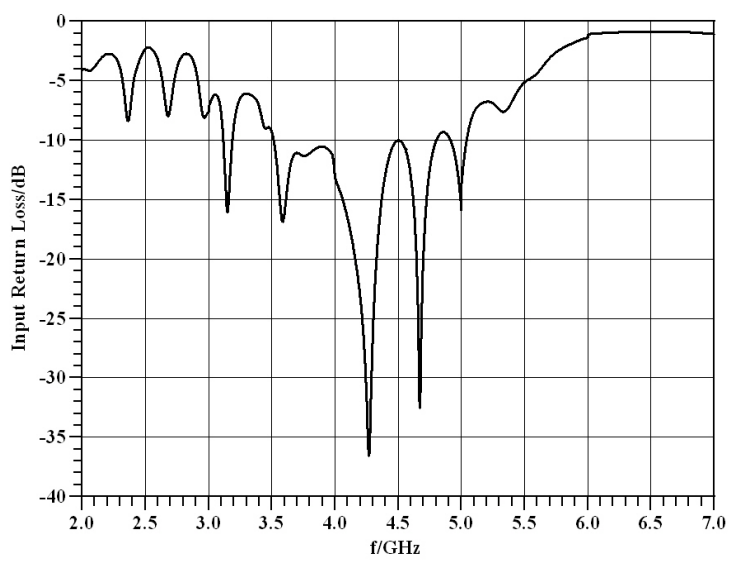

Figure 10. Input return loss performance of the UWB receiving antenna array shown in Fig. 1.

The normalized H-plane radiation patterns in the xy plane (c.f. Fig. 1) of the receiving antenna array are shown in Fig. 11 and Fig. 12. Again, radiation patterns are normalized to the peak gain of selected frequencies. For both vertical (Fig. 11) and horizontal polarizations (Fig. 12), radiation patterns are pointing upward (positive $\mathrm{x}$ direction) as expected. Multiple side lobes are created by single elements of the receiving antenna array. The co-polarized and cross-polarized patterns in the E-plane (xz plane) are shown in Fig. 13 and in Fig. 14.

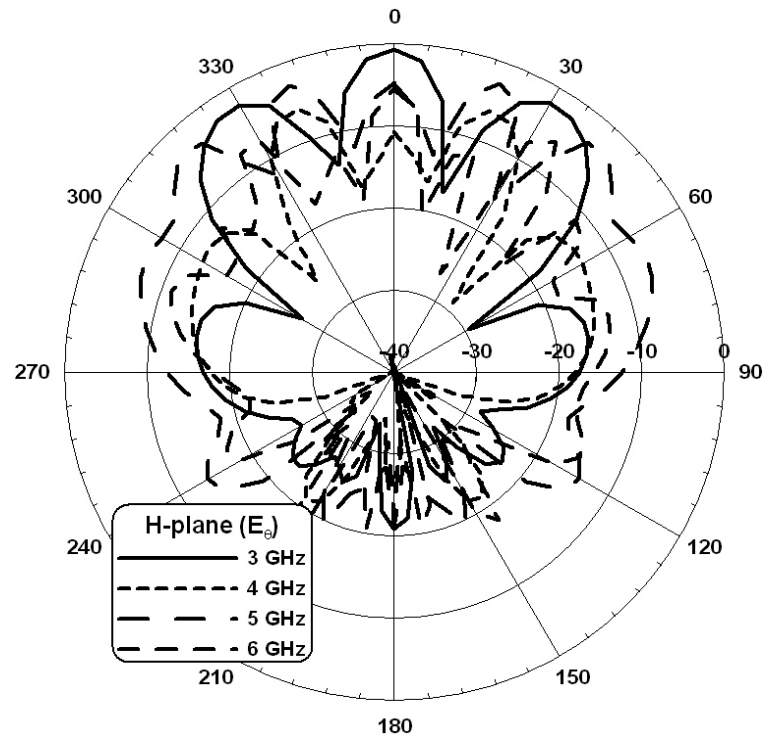

Figure 11. Normalized co-polarized H-plane (xy-plane) radiation patterns $\mathrm{E}_{\theta}(\pi / 2, \phi)$ of the UWB receiving antenna array for various frequencies.

\section{Maximum Gain}

Fig. 15 shows the maximum gain in the frequency range from $3 \mathrm{GHz}$ to $7 \mathrm{GHz}$ for both UWB transmitting antenna and UWB receiving antenna array.

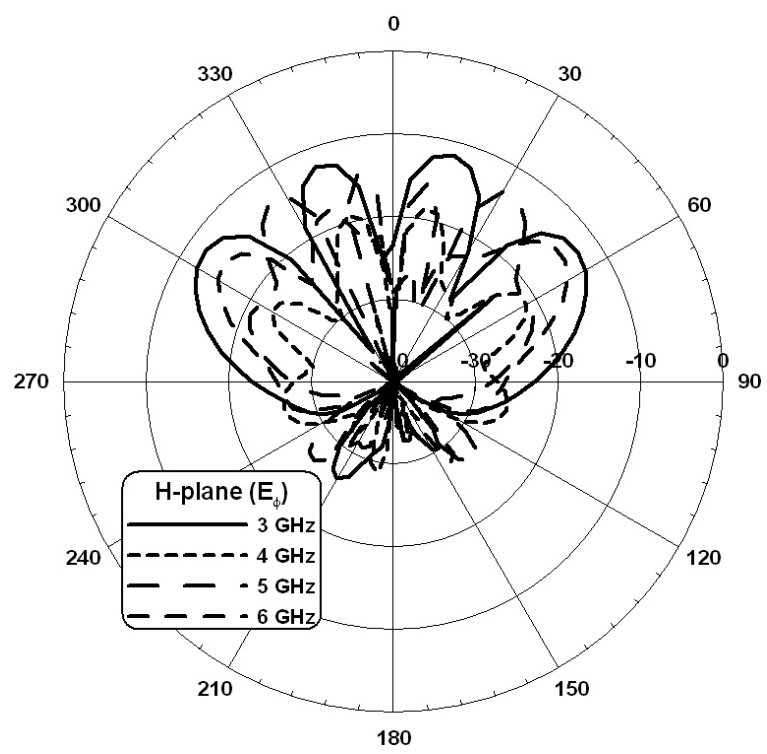

Figure 12. Normalized cross-polarized H-plane (xy-plane) radiation patterns $\mathrm{E}_{\phi}(\pi / 2, \phi)$ of the UWB receiving antenna array for various frequencies.

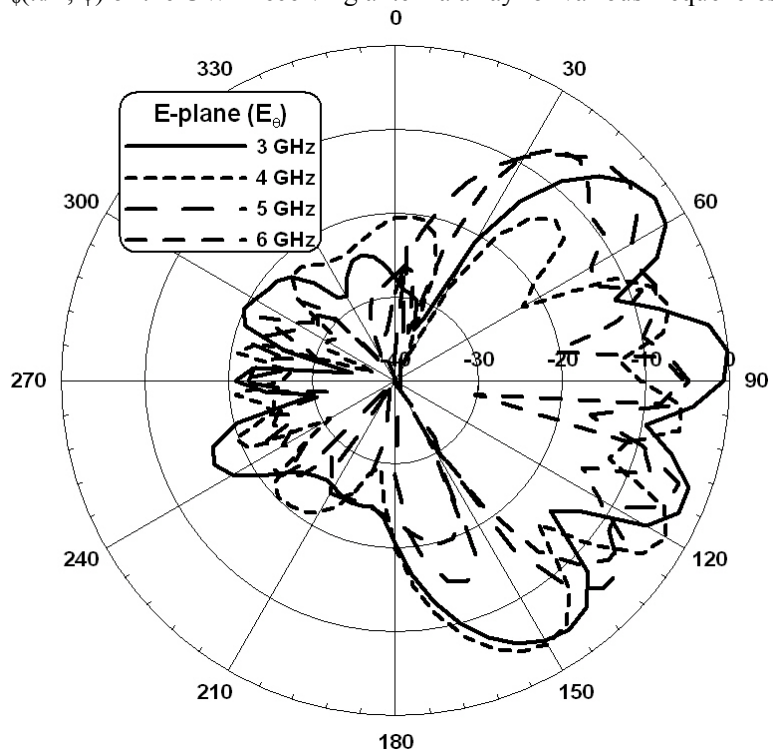

Figure 13. Normalized co-polarized E-plane (xz-plane) radiation patterns $\mathrm{E}_{\theta}(\theta, 0)$ of the UWB receiving antenna array for various frequencies.

\section{CONCLUSIONS}

The proposed ultra-wideband printed-circuit array antenna in coplanar waveguide technology presents a viable option for an UWB vital signs monitoring system. This antenna structure operates in both transmit mode $(3.4-5.9 \mathrm{GHz})$ and receive mode $(3.5-4.8 \mathrm{GHz})$. For the transmitting antenna, the input return loss is designed to increase the bandwidth of the generated UWB signal. As for the receiving antenna array, the input return loss is designed to only receive signals within the operating band of the generated UWB signal $(3.74-4.57$ $\mathrm{GHz}$ ). With the array structure for the receiving antenna, more antennas give more coverage, thus increase the overall signal strength of reflected signals. Radiation patterns for both transmitting antenna and receiving antenna array show expected characteristics and vary within acceptable margins over the entire frequency range. 


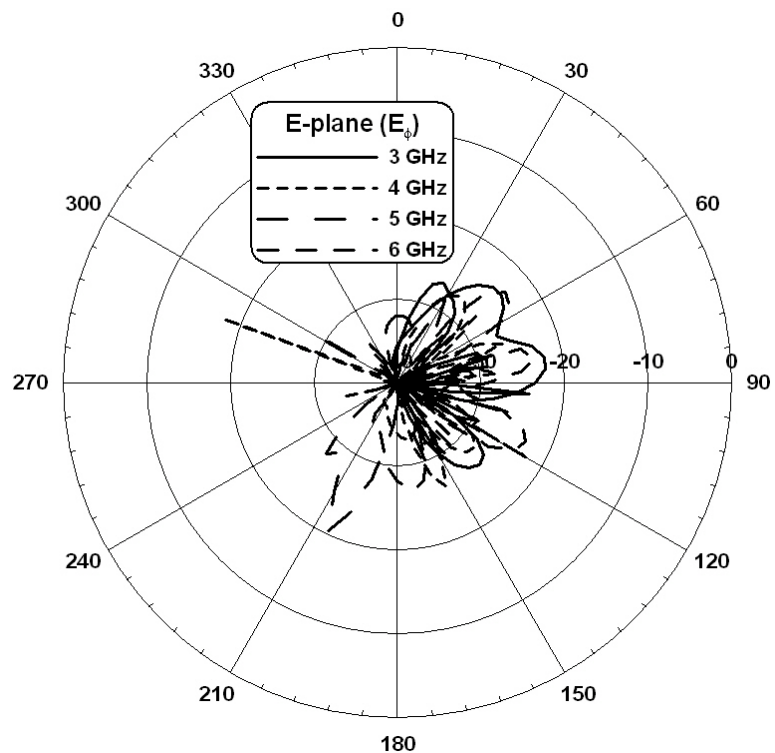

Figure 14. Normalized cross-polarized E-plane (xz-plane) radiation patterns $E_{\phi}(\theta, 0)$ of the UWB receiving antenna array for various frequencies.

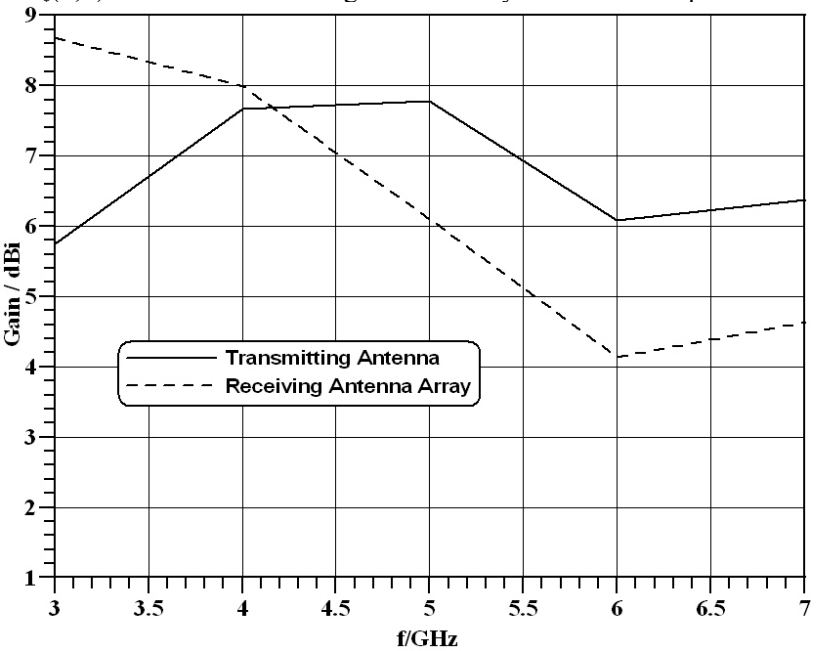

Figure 15. Maximum gain of the UWB antenna structure shown in Fig. 1

\section{REFERENCES}

[1] I. Immoreev and T-H Tao "UWB radar for patient monitoring," IEEE Aerospace and Electronic Systems Magazine, November, 2008.

[2] I. Immoreev, S. Samkov, and T-H Tao "Short- distance ultra-wideband radars. Theory and designing," RADAR 2004 International Conference on Radar Sytems.

[3] I. Immoreev, "Ultrawideband radars. Features and possibilities," Journal of Communications Technology and Electronics, 2009, Vol. 54, No. 1, pp. 1-26. Original Russian Text: I.Ya. Immoreev, 2009, published in Radiotekhnika i Elektronika, 2009, Vol. 54, No. 1, pp. 5-31.

[4] X. Chen, J. Liang, P. Li, L. Guo, C. C. Chiau and C. G. Parini, "Planar UWB monopole antennas," Proc. 2005 Asia-Pacific Microwave Conf., pp. 75-78, Dec. 2005.

[5] R. Chair, A. A. Kishk and K. F. Lee, "Ultrawide-band coplanar waveguide-fed rectangular slot antenna," IEEE Antennas Wireless Propagat. Lett., Vol. 3, pp. 227-229, No. 1, 2004.

[6] W. Wang, S.S. Zhong, and S.-B. Chen, "A novel wideband coplanar-fed monopole antenna," Microwave Opt. Technol. Lett., Vol. 43,pp. 50-52, 2004.

[7] T.-G. Ma and C.-H. Tseng, "An ultrawideband coplanar waveguide-fed tapered ring slot antenna," IEEE Trans. Antennas Propagat., Vol. 54, pp. 1105-1110, 2006.

[8] J.-S. Sun, Y.-C. Lee, and S.-C. Lin, "New design of a CPW-fed ultrawideband slot antenna," Microwave Opt. Technol. Lett., Vol. 49, pp. 561-564, 2007.

[9] D.-B. Lin, I.-T. Tang, and M.-Y. Tsou, "A compact UWB antenna with CPW-feed," Microwave Opt, Technol. Lett ., Vol. 49, pp. 564-567, 2007.

[10] Y. Lu, H.-J. Lam and J. Bornemann, "Coplanar printed-circuit antenna with band-rejection elements for ultra-wideband filtenna applications," IEEE AP-S Int. Symp. Dig., 526.4, 4 p., San Diego, USA, July 2008.

[11] Y. Lu, H.-J. Lam and J. Bornemann, "Coplanar UWB antenna with increased suppression characteristics," Microwave Opt. Technol. Lett., Vol. 50, pp. 3111-3114, Dec. 2008.

[12] A. Takacs, H. Aubert, P. Pons, T. Parra, R. Plana, and J. Graffeuil, "Miniature coplanar bandstop filter for Ka-band applications based on original resonant coupling irises topology," IEE Electronics Lett., Vol. 40, pp. 1274-1275, 2004.

[13] H.-J. Lam and J. Bornemann, "Ultra-wideband printed-circuit antenna in coplanar technology," IEEE EMC-S Int. Symp. Dig., TU-PM-1-7, pp. 14, Honolulu, USA, July 2007. 